\title{
Rosemary extract as a natural source of bioactive compounds
}

\author{
S.P.J. Namal Senanayake \\ Camlin Fine Sciences, CFS North America, LLC, Urbandale, Iowa, USA. Tel: 1-844-808-2063; E-mail: namal.senanayake@camlinfs.com \\ DOI: $10.31665 / \mathrm{JFB} .2018 .2140$ \\ Received: March 28, 2018; Revised received \& accepted: May 27, 2018 \\ Citation: Senanayake, S.P.J.N. (2018). Rosemary extract as a natural source of bioactive compounds. J. Food Bioact. 2: 51-57.
}

\begin{abstract}
Rosemary (Rosmarinus officinalis, L.) is an aromatic evergreen herb widely used around the globe as a spice/seasoning, flavoring agent and for medicinal applications. Rosemary extract derived from rosemary leaves contains several polyphenolic components which exhibit antioxidant properties, but the predominant active components are the phenolic diterpenes, namely, carnosic acid and carnosol. Over $90 \%$ antioxidant activity of rosemary extract is attributed primarily to high content of lipophilic antioxidant molecules such as carnosic acid and carnosol. In addition to antioxidant components, rosemary extract also contains some volatile compounds. Rosemary extract bioactive compounds are also responsible for their distinctive aroma, color and flavor attributes. Rosemary extract can be applied in a wide range of oils and fats, and lipid-containing foods to delay lipid oxidation and to enhance the shelf-life of various food, feed and pet food products. This review delineates the chemistry, antioxidant mechanism, volatile flavor compounds, regulatory position, food applications, and stability of rosemary extract in foods.
\end{abstract}

Keywords: Rosemary extract; Antioxidant; Phenolic diterpenes; Carnosic acid; Lipid oxidation.

\section{Introduction}

Rosemary (Rosmarinus officinalis, L.), a member of the Labiatae (Lamiaceae) family of plants, is a hardy evergreen perennial shrub that grows inherently in the Mediterranean region; however, it is cultivated around the globe due to its widespread use as a spice/ seasoning, flavoring agent and for medicinal purposes. In addition, due to its inherent antioxidant properties, rosemary extract has widely been used in the food industry for shelf-life enhancement of various food products. Rosemary herb is typically propagated via seeds, cuttings, layering or division of roots. Rosemary leaves can be harvested three to four times per year, depending on the geographical region and whether the harvest is for plant material or essential oil. The crop is cut frequently before flowering commences, as the dried product contains only leaves. For the fresh herbs market, the herb is cut frequently at a young stage as fresh shoots for use in various culinary and meat applications. It is only the leaves, or the extracts derived from the leaves that are commonly utilized as culinary herbs, food flavoring and naturally occurring antioxidants.

Commercially available rosemary extracts are produced by dry- ing and grinding rosemary leaves followed by extraction using various solvents such as ethanol, methanol, acetone, hexane, and water or using a mixture of solvents (Senanayake, 2013). The resulting mixture may be filtered to remove the biomass or the solid residue, followed by vacuum-based solvent evaporation, spray-drying and sieving to obtain a fine powdered extract. The further processing of the fine powder product may include deodorization, and/or decolorization using some food-grade excipients resulting in the production of the finished extract. Rosemary extract is also produced via supercritical fluid extraction. In this process, the dried rosemary leaves are extracted by means of supercritical $\mathrm{CO}_{2}$. Once the extraction is completed, the carbon dioxide fraction containing $\mathrm{CO}_{2}-$ soluble compounds is precipitated out to produce a raw extract. The further processing involves deodorization of the raw extract by supercritical $\mathrm{CO}_{2}$ to limit the content of essential oil and to achieve optimum concentration of the antioxidative components in the extract. Rosemary extract is commercially available as fine powdered products or as liquids after dilution/standardization with appropriate food-grade carriers ensuring consistent product quality. The choice of the rosemary extract product and the format depends on the type of food matrix where it is going to be applied. In addition to the antioxidative properties of rosemary extract, some studies have 


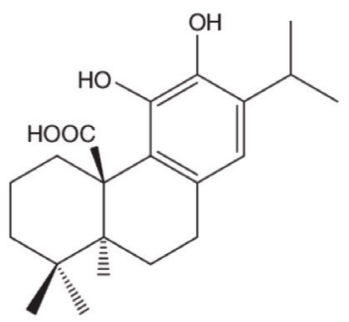

Carnosic acid

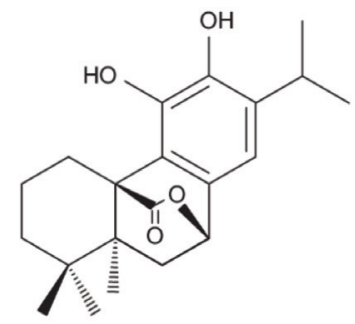

Carnosol
Figure 1. Phenolic diterpenes identified as principal antioxidative components of rosemary extract (Rosmarinus officinalis, L.).

revealed antimicrobial, anti-inflammatory, antitumorigenic, and antihyperglycemic effects of rosemary extract; however, the purpose of this article is to review the antioxidant properties of rosemary extract and its use as a shelf-life enhancer in food products.

\section{Rosemary phenolic compounds}

Rosemary extract contains diverse groups of polyphenol compounds including phenolic terpenes, flavonoids and some phenolic acids. The composition of rosemary extracts varies depending on the type of extraction procedure used. Although several antioxidant components have been identified, the most potent antioxidant compounds are the phenolic diterpenes, carnosic acid and carnosol, which account for over $90 \%$ of the antioxidant activity of rosemary extract. The structural formulas of carnosic acid and carnosol are shown in Figure 1. Other phenolic compounds and minor constituents found in rosemary extract such as rosmanol, epirosmanol, isorosmanol, rosmadial, rosmaridiphenol, and rosmariquinone also exert some antioxidative functions. In addition, triterpenes and triterpenic acids such as betulin, amyrin, betulinic acid, oleanic acid, and ursolic acid may also be present in rosemary extracts. Flavonoids present in rosemary extract include the flavones and flavonols such as apigenin, genkwanin, luteolin, hispidulin, rutin, kaempferol, naringin, hesperetin, apigenin-7-O-glucoside, and quercetin, among others. Phenolic acids reported in rosemary extract include rosmarinic acid, caffeic acid, chlorogenic acid, coumaric acid, $p$ coumaric acid, ferulic acid, vanillic acid, syringic acid, homovanillic acid and $p$-hydroxybenzoic acid, among many others.

\section{Antioxidant mechanism of action}

Lipid oxidation is one of the most critical aspects of quality deterioration in foods which also affect their functional properties, nutritional value, safety, color, flavor as well as consumer acceptability. The control of lipid oxidation in food remains to be a continuing challenge as most foods are made up of complex matrices. Oxidation of unsaturated lipids in food involves a free radical chain reaction that is generally initiated by the presence of ultraviolet and/or visible light, heat, air (oxygen) and prooxidant metal ions. Lipid oxidation produces various primary and secondary oxidation by-products that influence quality of food. The classical mechanism of autoxidation, free radical chain reaction, includes initiation, propagation, and termination steps. Lipid oxidation is initiated when a hydrogen atom from a site in the fatty acyl chain of unsaturated lipids is abstracted and lipid alkyl radicals are formed. The resultant lipid free radical reacts swiftly with triplet oxygen $\left({ }^{3} \mathrm{O}_{2}\right)$ and produces peroxyl radical as well as another reactive lipid free radical. The lipid peroxyl radical abstracts a hydrogen atom from another lipid molecule resulting in the formation of hydroperoxide $(\mathrm{LOOH})$ and a new lipid alkyl radical which can disseminate the oxidation chain reaction. The primary oxidation products, lipid hydroperoxides (ROOH), so produced are typically flavorless and odorless compounds. The decomposition of lipid hydroperoxides, which occurs in the presence of metals or at high temperature, leads to the formation of aldehydes, ketones, alcohols, acids, esters, furans, lactones and hydrocarbons. These compounds contribute to off-odors and off-flavors in food. The presence of naturally-occurring antioxidants such as those derived from rosemary extract may help control these degradative reactions.

Rosemary extract contains several bioactive compounds, which have proven to exert antioxidative properties, belonging mainly to the classes of phenolic acids, flavonoids, diterpenoids and triterpenes. Reminiscent of other phenolic antioxidants, the presence of phenolic diterpenes such as carnosic acid and its derivative carnosol in rosemary extract are capable of interfering with lipid oxidation by donating hydrogen atoms to lipid free radicals. Synthetic phenolic antioxidants such as butylated hydroxyanisole (BHA) or butylated hydroxytoluene (BHT) each have a single aromatic ring with one hydroxyl group that can serve as a hydrogen donor. Similarly, carnosic acid and carnosol each have a single aromatic ring; however, each molecule has two O-phenolic hydroxyl groups, located at $\mathrm{C}_{11}$ and $\mathrm{C}_{12}$ positions of catechol moiety, capable of donating hydrogen atoms $\left(\mathrm{H}^{*}\right)$ to lipid free radicals. Intrinsically, carnosic acid and carnosol have better antioxidant activity than BHA or BHT. In addition, the resultant radical intermediates of carnosic acid and carnosol are relatively stable due to resonance delocalization of unpaired electrons around the aromatic rings and lack of suitable sites in the phenoxyl radical for attack by the molecular oxygen. Moreover, the hydroxyl groups situated in carnosic acid and carnosol molecules have the ability to chelate prooxidant metal ions, and thus help delay lipid oxidation via a secondary mechanism. Research also suggests that rosemary phenolic diterpenes are capable of scavenging various types of free radicals including peroxyl, hydroxyl and DPPH radicals.

Rosemary phenolic diterpenes, carnosic acid and carnosol, can be further oxidized on exposure to elevated temperatures, oxygen, and light; however, their ability to delay lipid oxidation is maintained (Senanayake, 2013). When carnosic acid reacts with lipid free radicals, it converts into a carnosol molecule. Carnosol also reacts with lipid free radicals and converts into rosmanol. Hence, the principal decomposition products of carnosic acid are carnosol and rosmanol. Meanwhile, minor degradation products of carnosic acid is epirosmanol. It has been postulated that rosmanol continues the free radical scavenging process until it becomes galdosol (Fig. 2). Liu et al. (2013) studied the degradation pathway of carnosic acid in a methanolic solution through isolation and structural identification of its degradation products. They reported that carnosic acid can decompose into carnosic acid quinone in methanol. Carnosic acid quinone is the quinone intermediate of carnosic acid that can be reduced into carnosic acid or can be further converted into carnosol. Carnosol can further decompose to form epirosmanol, rosmanol, 7-methoxyrosmanol, and 7-methoxyepirosmanol. The latter three compounds were identified as the final degradation products of carnosic acid in methanol because they appeared to be rather stable in the study.

\section{Volatile flavor compounds}

In addition to active antioxidant components, rosemary extract contains several volatile components that are responsible for its flavor 
Free Radical

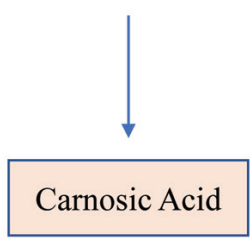

Free Radical

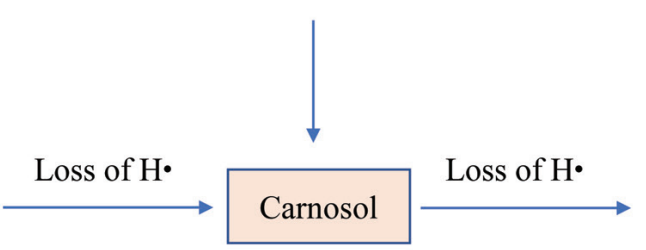

Free Radical

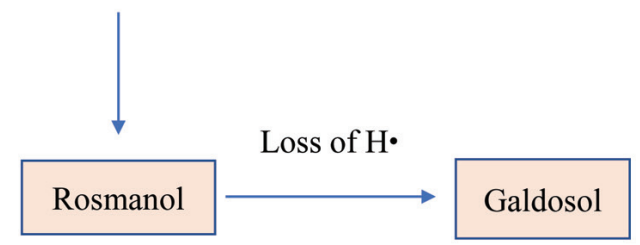

Figure 2. Oxidation cascade reactions of carnosic acid.

and odor attributes. The main volatile compounds found in rosemary extract essential oil fraction include camphor, borneol, bornyl acetate, eucalyptol (1,8-cineol), verbenone, $\alpha$-terpineol, $\alpha$-pinene and limonene, among others (Senanayake, 2013). The essential oil fraction of rosemary extract is a colorless or pale-yellow liquid that contains these volatile compounds. The essential oils derived from rosemary herb are reported to exhibit antibacterial, antifungal and anticancer properties. The main compounds responsible for the antimicrobial activity are camphor, bornyl acetate, $\alpha$-pinene and eucalyptol. Bozin et al. (2007) investigated the antibacterial activity of essential oils derived from rosemary and sage against a number of food spoilage microorganisms. They concluded that the essential oils of both rosemary and sage were effective against Escherichia coli, Salmonella typhi, S. enteritidis, and Shigella sonei. In addition, rosemary essential oil exhibited some antifungal activity. Similar results were obtained by Fu et al. (2007) for the antibacterial activity of essential oils from rosemary and sage against some bacterial strains. Although rosemary extract contains both volatile compounds and antioxidant components, it may be produced such that its antioxidant functionality is enhanced. This can be done via steam distillation to remove some of the volatile oil fraction from the extract. However, residual quantities of volatile flavor compounds may remain in the final extract which may exert some flavor effects.

\section{Antioxidant properties}

Of the natural antioxidants, rosemary extract has been widely rec- ognized as one of the spices/seasoning that exhibits high antioxidant activity in numerous food applications. According to Moreno et al. (2006), both antioxidant and antimicrobial activities of rosemary extract are linked to its polyphenol composition. A good correlation existed between the antioxidant activities and total phenol content in the extracts. Although rosemary extract showed a high radical scavenging activity, a different efficacy as antimicrobial agent was observed. Methanol extract containing $30 \%$ of carnosic acid, $16 \%$ of carnosol and $5 \%$ of rosmarinic acid was the most effective antimicrobial against gram positive bacteria, gram negative bacteria and yeast. By contrast, water extract containing only $15 \%$ of rosmarinic acid showed a narrow activity range. Therefore, their results suggest that the antimicrobial activity of rosemary extracts was associated with their specific phenolic composition. Carnosic acid and rosmarinic acid may be the main bioactive antimicrobial compounds present in rosemary extracts.

Rosemary extract has been well recognized in the food industry as a natural alternative to synthetic antioxidants such as BHA or BHT. The main characteristics of commercially available rosemary extracts are listed in Table 1. Rosemary extracts, which are considered Generally Recognized as Safe (GRAS), have diverse applications in various food and beverage product categories (Table 2). They have been widely used in several food products such as meat, poultry and seafood, dressings and sauces, potato chips, and baked goods. For beverage applications, rosemary extracts containing hydrophilic antioxidant molecules (i.e., rosmarinic acid) are commonly used. In most food applications, the actual usage rates of rosemary extract will vary depending on the total fat content of the food as well as the nature of the fat and intended use

Table 1. Main features and benefits of commercially available rosemary extracts

Natural alternative to synthetic antioxidants

Clean label option

Ease of handling, distribution and application

Standardized antioxidant activity

Low odor and flavor intensity

Relative heat stability

Availability of optimized tailored solutions

Shelf-life extension in food

Reduce formation of rancid flavors and off-odors in food

Promotes longer food product freshness by protecting flavor, aroma, and/or color

Minimize food product loss

Increase consumer satisfaction

Widespread application prospects in food

Protection of brand image 


\section{Table 2. Applications of rosemary extract in food}

Vegetable oils, animal fats, marine oils, and frying oils

Salad dressings, and mayonnaise

Margarines, and fat spreads

Potato products

Fresh meat, poultry, and seafood products

Cooked meats, poultry, and seafood products

Cereals, bakery products, and confections

Snack foods, and nuts

Pet food, animal protein meals, and animal feed

Beverages

of the finished food product.

Rosemary extract is quite effective in improving oxidative stability of a wide array of edible oils and fats. Based on the relative resistance to oxidation of various oils and fats that can be monitored using the Oxidative Stability Instrument (OSI), a Protection Factor (PF) can be calculated for specific dosage of rosemary extract. Protection factor is the ratio of the number of OSI hours for the treated oil or fat to the OSI hours for the respective untreated control. Protection factor is an indication of the extent of stability improvement in oil or fat obtained with rosemary extract. Typically, as the PF increased, the oxidative stability of the oil or fat increased (Table 3). The PF can also be used to predict shelf life extension of oils. The shelf life extension is computed as follows:

$$
\text { Life Extension }(\%)=(P F-1) \times 100
$$

In a storage stability test conducted by Camlin Fine Sciences laboratories, the performance of rosemary extract-ascorbyl palmitate combination was compared to commonly used synthetic antioxidants such as BHT and tertiary butylhydroquinone (TBHQ) in soybean oil. The oil samples treated with these antioxidants were stored at $40{ }^{\circ} \mathrm{C}$ for up to 65 days. Progression of oxidation was monitored by measuring changes in peroxide values of oil samples. Rosemary extract-ascorbyl palmitate combination exhibited excellent antioxidant activity and its efficacy was higher than BHT, but slightly less than that of TBHQ (Fig. 3). Frankel et al. (1996) measured the antioxidant activity of a commercial rosemary extract and the active constituents carnosol, carnosic acid, and rosmarinic acid, in inhibiting the formation and decomposition of hydroper- oxides in tocopherol-stripped corn oil and in the corresponding corn oil-in-water emulsions. In bulk corn oil, the rosemary extract, carnosic acid, rosmarinic acid, and $\alpha$-tocopherol were significantly more active than carnosol. In contrast, in corn oil emulsion, the rosemary compounds were less active than in bulk oil, and the rosemary extract, carnosic acid, carnosol, and $\alpha$-tocopherol were more active than rosmarinic acid. The decreased antioxidant activity of the polar hydrophilic rosemary compounds in the emulsion system may be explained by their interfacial partitioning into the water, thus becoming less protective than in the bulk oil system.

Frying oils provide an important application area for rosemary extract due to their high susceptibility to lipid oxidation. A study conducted by Casarotti and Jorge (2014) examined the antioxidant activity of lyophilized rosemary extract added to soybean oil, subjected to thermal oxidation and its synergistic effect with the synthetic antioxidant TBHQ. Soybean oil samples with no antioxidant added, 3,000 ppm rosemary extract, $50 \mathrm{ppm}$ TBHQ, and a mixture of two antioxidants were heated to $180^{\circ} \mathrm{C}$ for $20 \mathrm{~h}$. The addition of rosemary extract increased oxidative stability and resulted in a lower formation of total polar compounds and a higher retention of tocopherols. The rosemary extract treatment showed the highest retention of polyunsaturated fatty acids after $20 \mathrm{~h}$. There was no synergy between TBHQ and rosemary extract in preventing oxidation of soybean oil. Rosemary extract showed a higher antioxidant potential when compared with TBHQ. Lalas and Dourtoglou (2003) evaluated the effect of rosemary extract on the stability of oil used for frying potato chips. Sliced potatoes were fried intermittently in soybean oil containing rosemary extract. The conditions used for frying were $185^{\circ} \mathrm{C}$ for 90 seconds. The induction period of oils, as measured by Rancimat, decreased as frying progressed. The reduction in the induction period was higher in the oil free of rosemary extract, and the chips were much darker in color. The oil containing the extract showed greater antioxidant activity, and reduced darkening and rancidity. Potato chips fried in the oil with added rosemary extract were more acceptable than chips fried in oil containing no extract until the last frying. In a study by Cordeiro et al. (2013), the antioxidant effect of the ethanolic extract of rosemary on the oxidative stability of edible vegetable oils was investigated by means of pressurized differential scanning calorimetry (PDSC) and oven test techniques. The rosemary extract (at 2,000 ppm) and TBHQ (at 100 and $200 \mathrm{ppm}$ levels) were added to samples of sunflower oil, corn oil, and soybean oil. The thermogravimetric analysis showed that the rosemary extract was stable at the frying temperature of the oils. The results of the oxidative stability demonstrated that the rosemary extract displayed a more effective protective action in the PDSC technique, when compared with the TBHQ. Chammem et al. (2015) studied the effect of rose-

Table 3. Antioxidant activity of rosemary extract in selected oils and fats

\begin{tabular}{llll}
\hline Sample & Oil Stability Index (hours) & Protection Factor (PF) & Shelf-Life Extension (\%) \\
\hline Soybean Oil with no Additives & 5.2 & - & - \\
Rosemary Extract 1 (2,000 ppm) & 7.9 & 1.5 & 53.9 \\
Rosemary Extract 2 (2,000 ppm) & 9.2 & 1.8 & 79.1 \\
Sunflower Oil with no Additives & 6.3 & - & - \\
Rosemary Extract 1 (2,000 ppm) & 8.2 & 1.3 & 30.0 \\
Rosemary Extract 2 (2,000 ppm) & 9.3 & 1.5 & 48.0 \\
Chicken Fat with no Additives & 5 & - & - \\
Rosemary Extract 1 (2,000 ppm) & 16.1 & 3.2 & 222.5 \\
Rosemary Extract 2 (2,000 ppm) & 18.5 & 3.7 & 269.0 \\
\hline
\end{tabular}




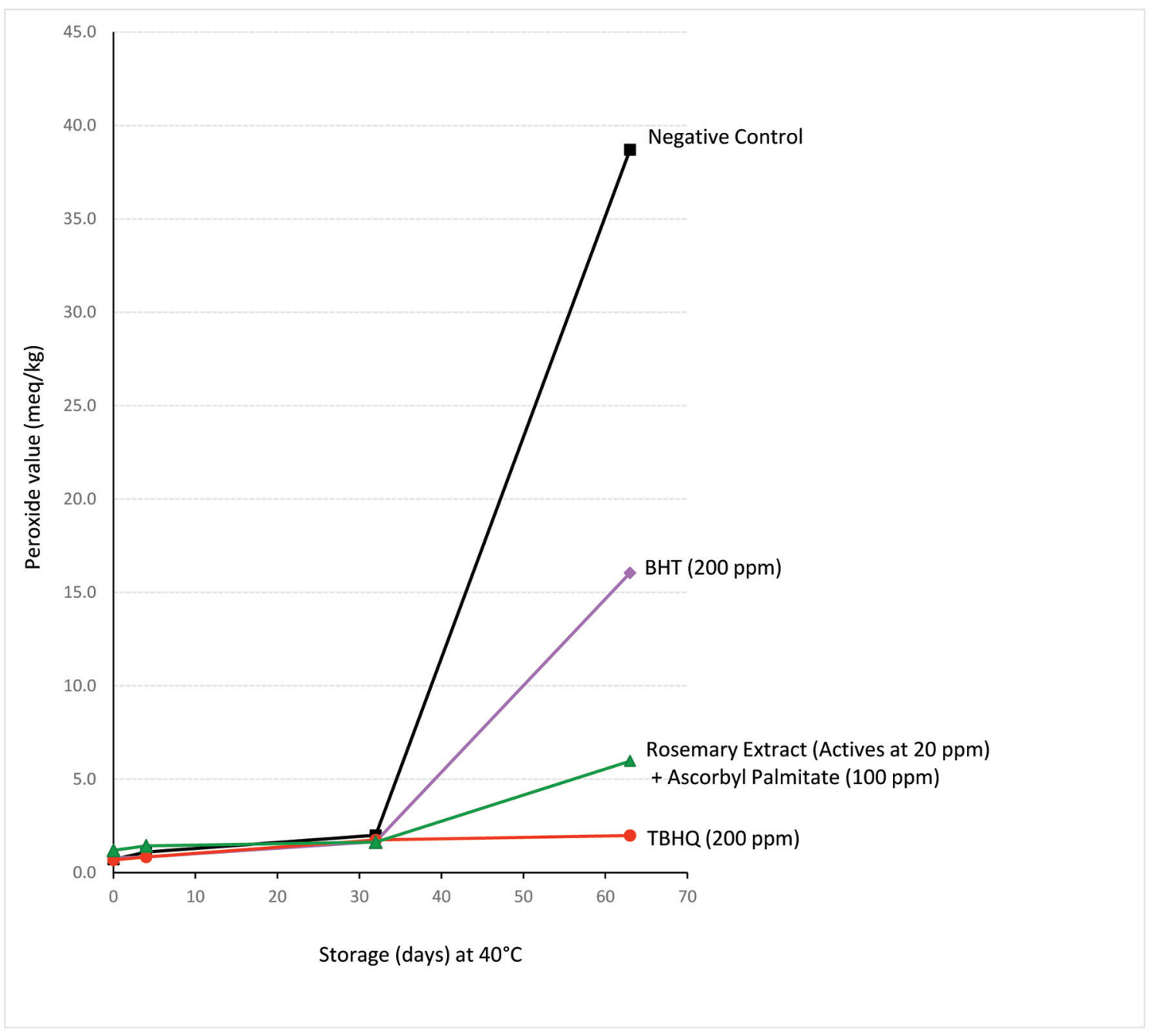

Figure 3. Peroxide values of soybean oil treated with antioxidants during storage at $40^{\circ} \mathrm{C}$.

mary extract on the stability of frying oil and on sensory quality of fried potatoes. A mixture of soybean and sunflower oils in equal proportions and containing $800 \mathrm{ppm}$ of rosemary extract was evaluated for thermal oxidation at $180{ }^{\circ} \mathrm{C}$ for $30 \mathrm{~h}$. This evaluation used an oil mixture without antioxidant for comparison. Results showed a significant difference $(p<0.05)$ between oils according to the measured parameters especially at the end of treatment. The addition of the rosemary extract in the mixture of soybean and sunflower oils reduce the peroxide value by $38 \%$ after $30 \mathrm{~h}$ of heating. Sensory analysis carried out showed that the fried potato prepared in oil with rosemary extract had the best crispiness and taste until the fifteenth frying. The oil with rosemary extract showed an enhanced stability and therefore the best quality compared to the oil without rosemary extract.

Rosemary extract is normally added to various meat, poultry and seafood products to prevent lipid oxidation, retard development of off-flavors, and improve their color stabilities. In heatprocessed meat products, including cooked, refrigerated and precooked, as well as frozen meat products, lipid oxidation leads to development of warmed-over flavor (WOF). This undesirable flavor is commonly characterized as "stale", "cardboard-like", "painty" or rancid". WOF, which originates due to oxidation of meat fat, can also occur in raw meat products that have been exposed to membrane disruption. Primary oxidation products so produced decomposed into secondary oxidation products such as pentanal, hexanal and 2,4-decadienal and detected as WOF. This meat flavor deterioration can be effectively retarded using rosemary extract. Thongtan et al. (2005) investigated the effect of rosemary extract on lipid oxidation in frozen, precooked beef patties stored at -18 ${ }^{\circ} \mathrm{C}$ for 12 weeks. Samples were analyzed for peroxide value (PV) levels, 2-thiobarbituric acid (TBA) values and sensory characteristics on 0 day and after 4,8 , and 12 weeks. Samples with rosemary extract showed lower PV and TBA values. Sensory evaluations of precooked patties with rosemary extract were not significantly different in scores on color, odor, appearance, texture and overall acceptability. Samples with rosemary extract reported a significantly higher liking score for flavor. In a study by Keokamnerd et al. (2008), four commercial rosemary extract preparations were added to ground chicken thigh meat at the recommended levels of the manufacturer then packaged in $80 \% \mathrm{O}_{2}-20 \% \mathrm{CO}_{2}$ modified atmosphere trays. The rosemary preparations differed in oil and water solubility, dispersion properties, or both. The addition of rosemary extract to ground chicken had an overall positive effect on raw meat appearance during storage and on the cooked meat flavor. 
Meanwhile, no effect on bacterial growth was observed due to the addition of rosemary extract. However, oxidation was slowed down in meat with added rosemary extract as indicated by lower TBA values, lower hexanal contents, and sensory scores. The color (redness of meat) was more stable in meat with added rosemary extract compared with meat without rosemary extract, as reflected in redness $\left(\mathrm{a}^{*}\right)$ values, hue angles, and visual scores. Of the four rosemary extract preparations tested, the oil-soluble, most concentrated preparation was most effective in maintaining meat quality compared with the other three types tested. In another study, Azizkhani and Tooryan (2015) evaluated the effects of extracts of rosemary, mint and mixed tocopherols on microbiological parameters and lipid oxidation in beef sausages stored for 25 days at $4{ }^{\circ} \mathrm{C}$. The addition of rosemary extract resulted in significant inhibition of microbial growth and the lowest microbial counts were obtained in samples containing both rosemary and mint extracts, indicating a possible synergistic effect. Rosemary and its combination with mixed tocopherols or mint extract showed the greatest antioxidative effect. The shelf-life of samples containing rosemary extract was almost doubled compared with the remaining samples and the best antimicrobial and antioxidative effects were obtained from the combination of rosemary with mint extract. A recent study was undertaken by Rajeev et al. (2017) to evaluate the efficacy of a deflavored and decolorized rosemary extract for preservation of naturally colored fresh beef patties. Oxidative rancidity of meat and color degradation of paprika oleoresin were exploited as model systems to compare the antioxidant performance of rosemary extract with BHA. The results showed similar efficacy for 3\% carnosic acid extract and BHA, with further enhancement in efficacy with respect to the carnosic acid content. A synergetic antioxidant effect of carnosol and carnosic acid was also noticed to an extent of $1: 1(\mathrm{w} / \mathrm{w})$ ratio, and further increase in carnosol content showed no improvement in the antioxidant efficacy. Ultimately, a mixture of paprika and rosemary extract containing carnosic acid and carnosol at 1:1 (w/w) ratio was successfully applied to fresh beef patties to produce naturally colored meat products suitable for storage at $4{ }^{\circ} \mathrm{C}$.

The use of rosemary extract in animal feed has been documented by several investigators. Patent Application WO2013110845 A1 describes the use of rosemary extract in animal feed which was intended for feeding ruminants (Patent WO 2013110845 A1, 2013). This invention relates to rosemary extract comprising phenolic diterpenes at a concentration ratio of carnosic acid to carnosol between 0.7 and 1.2. The invention highlights the importance of the relationship between the concentration of carnosic acid and carnosol in rosemary extract when added to the feed for animal nutrition, as it is essential to ensure a proper bioavailability of both active components and thereby ensuring an effective improvement in the quality and shelf-life of meats. The study by Nieto et al. (2010) evaluated the effect of including distilled rosemary leaf in the diet of pregnant ewes on subsequent lamb meat quality. Thirtysix ewes were randomly assigned to three homogeneous groups. One group was fed a basal diet as control while the diet of the other two groups was modified by substituting 10 and $20 \%$ of the basal diet with a pellet made from $50 \%$ barley and $50 \%$ of distilled rosemary leaves. Fresh lamb meat samples produced were stored under modified atmosphere packaged $\left(70 \% \mathrm{O}_{2}: 30 \% \mathrm{CO}_{2}\right)$ conditions and evaluated for meat spoilage, oxidation products, color and the sensory characteristics during storage. In general, distilled rosemary leaves supplemented diets had higher color $a^{*}$ values (redness), and lower TBARS and lower rancid odor than the control samples. In addition, the total viable count was lower in meat from the diet fed with distilled rosemary leaves as compared to the control sample. In a study by Ortuño et al. (2014), the use of dietary rosemary extract at low doses was proposed as a nutritional strategy to improve meat preservation. Lamb diet was supplemented with 0,200 or $400 \mathrm{ppm}$ of dietary rosemary extract containing carnosic acid and carnosol (at 1:1, w/w) during the fattening stage. Meat quality was evaluated in lamb fillets packed under protective atmosphere and kept under retail conditions for up to 14 days. The effects of diet and storage time were determined on different physico-chemical (color L*, a*, b* values, $\mathrm{pH}$, TBARS, protein oxidation and lipid oxidation volatiles), microbial (total viable and psychrophilic bacteria, enterobacteriaceae, molds and yeasts) and sensory (appearance and odor) characteristics of the meat. The antioxidant and antimicrobial effects of dietary rosemary extract on meat were demonstrated. Dietary rosemary extract delayed lean and fat discoloration, lipid oxidation, odor deterioration and microbial spoilage, extending the shelf life of fillets from around 9 to 13 days. Both dietary rosemary extract doses provided similar shelf life extension in meats.

\section{Regulatory insight and labelling}

Rosemary extract is widely recognized globally for food use. In the United States, extract of the plant rosemary (Rosmarinus officinalis, L.) is approved as Generally Recognized as Safe (GRAS) for use in foods as natural seasoning and flavoring when used in accordance with Good Manufacturing Practice (GMP) as published in Title 21 of the Code of Federal Regulations in Part 182.10 (U.S. Food and Drug Administration, 2017). As for labelling purposes in the United States, it may be declared on food labels as rosemary extract, spice extract, or natural flavoring. As noted previously, rosemary extract exhibit both the antioxidant and flavoring properties. In many instances, both functions are utilized within the food; however, in European Union (EU) some specific rosemary extracts are marketed primarily for their antioxidant properties because of the EU legislation permitting the use of specific rosemary extracts as approved food additives (antioxidants). Food additives are functional substances that are not consumed as a food by themselves nor are they part of the main list of ingredients, whether they have nutritional value or not, but are intentionally added to food to aid in its processing or to improve the appearance, texture, consistency, color, flavor, odor or shelflife. As published in Directives 2010/67/EU and 2010/69/EU, it indicates the specific criteria under which some rosemary extracts may be used and labelled in some foods as a food additive of the antioxidant class. As such, in the food labels it should be appeared as "Antioxidant: Extract of Rosemary" or as "E-392" (antioxidants belong to the 300 series of the list of additives, with rosemary extract appearing on position 92). Per Directive 2010/69/ $\mathrm{EU}$, it is the total amount of carnosic acid and carnosol which needs to be considered rather than the use level of the whole rosemary extract. Hence, the levels of active antioxidant compounds within the rosemary extract should be determined. Based on these criteria, rosemary extracts containing carnosic acid and carnosol must be produced by one of four precise extraction methods (using acetone, supercritical $\mathrm{CO}_{2}$, ethanol, or a mixture of ethanolhexane). The extracts so produced are characterized by their carnosic acid and carnosol content, as well as their specified and limited flavor content. The volatile components mainly contribute to the characteristic flavor and aroma of rosemary extract were listed as borneol, bornyl acetate, camphor, 1,8-cineol, and verbenone. The percent weight ratio of carnosic acid plus carnosol to total weight percent of reference key volatiles is expected to be $\geq$ 15. Thus, if rosemary extract is added primarily as an antioxidant, 
the specifications laid down in the EU legislation should be met. As there is no intent with this legislation to eliminate rosemary as a flavoring material, some rosemary extracts may still be used as natural flavorings in food.

\section{References}

Azizkhani, M., and Tooryan, F. (2015). Antioxidant and antimicrobial activities of rosemary extract, mint extract and a mixture of tocopherols in beef sausage during storage at 4C. J. Food Saf. 35(1): 128-136.

Bozin, B., Mimica-Dukic, N., Samojlik, I., and Jovin, E. (2007). Antimicrobial and antioxidant properties of rosemary and sage (Rosmarinus of ficinalis L. and Salvia officinalis L., Lamiaceae) essential oils. J. Agric. Food Chem. 55(19): 879-7885.

Casarotti, S.N., and Jorge, N. (2014). Antioxidant activity of rosemary extract in soybean oil under thermoxidation. J. Food Process Preserv. 38: 136-145.

Chammem, N., Saoudi, S., Sifaoui, I., Sifi, S., Person, M., Abderraba, M., Moussa, F., and Hamdi, M. (2015). Improvement of vegetable oils quality in frying conditions by adding rosemary extract. Ind. Crops Prod. 74(15): 592-599.

Cordeiro, M.T.M., Medeiros, M.L., Santos, N.A., Soledade, L.E.B., Pontes, L.F.B.L., Souza, A.L., Queiroz, N., and Souza, A.G. (2013). Rosemary (Rosmarinus officinalis L.) extract. J. Therm. Anal. Calorim. 113: 889895.

Frankel, E.N., Huang, S.-W., Aeschbach, R., and Prior, E. (1996). Antioxidant activity of a rosemary extract and its constituents, carnosic acid, carnosol, and rosmarinic acid, in bulk oil and oil-in-water emulsion. J. Agric. Food Chem. 44(1): 131-135.

Fu, Y., Zu, Y., Chen, L., Shi, X., Wang, Z., Sun, S., and Efferth, T. (2007). Antimicrobial activity of clove and rosemary essential oils alone and in combination. Phytother. Res. 21: 989-994.

Keokamnerd, T., Acton, J.C., Han, I.Y., and Dawson, P.L. (2008). Effect of commercial rosemary oleoresin preparations on ground chicken thigh meat quality packaged in a high-oxygen atmosphere. Poultry Sci. 87(1): 170-179.

Lalas, S., and Dourtoglou, V. (2003). Use of rosemary extract in preventing oxidation during deep-fat frying of potato chips. J Am. Oil Chem. Soc. 80: 579-583.

Liu, X., Du, J., Ou, Y., Xu, H., Chen, X., Zhou, A., He, L., and Cao, Y. (2013) Degradation pathway of carnosic acid in methanol solution through isolation and structural identification of its degradation products. Eur. Food Res. Technol. 237(4): 617-626.

Moreno, S., Scheyer, T., Romano, C.S., and Vojnov, A.A. (2006). Antioxidant and antimicrobial activities of rosemary extracts linked to their polyphenol composition. Free Radic. Res. 40(2): 223-231.

Nieto, G., Díaz, P., Bañón, S., and Garrido, M.D. (2010). Dietary administration of ewe diets with a distillate from rosemary leaves (Rosmarinus officinalis L.): Influence on lamb meat quality. Meat Sci. 84(1): 23-29.

Ortuño, J., Serrano, R., Jordán, M.J., and Bañón, S. (2014). Shelf life of meat from lambs given essential oil-free rosemary extract containing carnosic acid plus carnosol at 200 or $400 \mathrm{mg} \mathrm{kg}^{-1}$. Meat Sci. 96(4): 1452-1459.

Rajeev, P.S., Johannah, N.M., Gopakumar, G., Maliakel, B., and Krishnakumar, I.M. (2017). Optimization of antioxidant efficacy of a deflavored and decolorized rosemary extract: Effect of carnosol content on the oxidative stability of paprika colored beef patties. J. Food Sci. Technol. 54: 1665-1677.

Senanayake, S.P.J.N. (2013). Rosemary and green tea extracts as natural antioxidants: Chemistry, technology, and applications. In: Logan, A., Nienabar, U. and Pan, X. (Ed.). Lipid oxidation: Challenges in food systems. AOCS Press, Urbana, IL, pp. 439-456.

Thongtan, K., Toma, R.B., Reiboldt, W., and Daoud, A.Z. (2005). Effect of rosemary extract on lipid oxidation and sensory evaluation of frozen, precooked beef patties. Foodserv. Res. Intl. 16(3-4): 93-104.

U.S. Food and Drug Administration (2017). Code of Federal Regulations, Title 21, Volume 3. Office of the Federal Register. U.S. Government Printing Office, Washington, DC.

Patent WO 2013110845 A1 (2013). Rosemary extract and use thereof in animal feed, August 2013. 